\title{
On sliding mode control design for UAV using realistic aerodynamic coefficients
}

\author{
Gabriele Perozzi, Denis Efimov, Jean-Marc Biannic, Laurent Planckaert, Patricia Coton
}

\begin{abstract}
The goal of this paper is to design a control of mini quadrotor under wind perturbations. Taking into account a detailed unmanned aerial vehicle (UAV) model, the aim is to find a sliding mode control law minimizing the impact of the wind field on UAV dynamics. For this purpose an aerodynamic modelization of external disturbance is introduced. After that, upper bounds of these disturbances are computed. Lastly, the sliding mode altitude and attitude controls are designed. The peculiarity of the considered case is that the disturbance upper bounds depend on the control amplitude itself (i.e. the system is nonlinear in control), which leads to a new procedure for the control tuning presented in the paper. The results of numeric experiments demonstrate the effectiveness of the obtained controls, and the comparisons with a conventional first order sliding mode control, having the function sign proportional to a constant gain, and a recent quasi-continuous sliding mode control are also given.
\end{abstract}

\section{INTRODUCTION}

Autonomous UAVs are increasingly popular platforms in last decades. They are useful for many applications such as monitoring, inspection, and other actions especially in urban areas or nearby buildings and interiors. These machines are often required to move in unfamiliar environments in terms of geography and in terms of the aerological conditions. In addition, the low mass of such units (comparing to the forces generated by the air disturbances) reduces significantly the domain of stable flight, then additional constraints have to be considered in the control design. Thus, it appears inevitable that, if we want to let UAVs operate in urban environments, inside turbulent airflow patterns for which accurate prediction is not possible with limited resources, we need to focus on detailed aerodynamic models and sophisticated control laws. This paper is part of a project at Onera lab of Lille which aims to use the drone itself as wind sensor in a navigation process usable in windy urban flight scenario. The objective is to build a robust altitude and attitude nonlinear controller for a small UAV with a limited knowledge of the wind perturbation. The aerodynamic model described in this article can be used to estimate wind speed as in [1], the estimated values can be used in the control to properly adapt the gains on-line.

G. Perozzi, L. Planckaert and P. Coton are with ONERA - The French Aerospace Lab, DAAA, 5 Bd Paul Painlevé BP 2126159014 Lille Cedex France.

D. Efimov is with Inria, Non-A team, Parc Scientifique de la Haute Borne, 40 avenue Halley, Bât. A, Park Plaza, 59650 Villeneuve d'Ascq France.

JM. Biannic is with ONERA - The French Aerospace Lab, DTIS, BP74025 2 avenue Edouard Belin 31055 Toulouse Cedex 4 France.

This work was funded by Hauts-de-France region and ONERA.

\section{A. State of the Art}

In literature, the quadrotor physical models with different grade of complexity are presented and used for different tasks. For example, to mention the most relevant in our study, [2] considers aerodynamic moments and forces varying in time affected by the perturbations, then linear PID controllers are applied. The work [3] presents more deeply the dynamic equations than the previous ones, but it does not take into account the wind effects.

There exist many control design techniques to counteract the effects of wind perturbations on flight of small UAVs, among which sliding mode control (SMC) plays a keyrole. The main reason consists in its excellent robustness properties against matched perturbations and measurement noises, and finite-time rate of convergence also. Many methods have been proposed in literature, [4] illustrates all the principal SMCs with their relative sliding surfaces and Lyapunov functions. Hundreds of articles have applied SMC to quadrotors in order to solve the position and the attitude tracking problems ensuring robustness against external disturbances. Just to take some of them, a chattering-free SMC was proposed in [5], replacing the sign function with a high-slope saturation function. A SMC based on Backstepping approach was introduced in [6], and an enhanced version was proposed in [7] using fuzzy logic. The works [8] and [9] use an algorithm based on the second order SMC technique known as SuperTwisting Algorithm. Second order SMC was also proposed in [10], and [11] uses a high-order SM observer as an estimator of the effect of the external disturbances such as wind and noise. Integral SMC were proposed in [12], and an adaptive SMC was developed in [13].

In this paper a robust nonlinear SMC law design is described, which considers realistic assumptions on external disturbances of quadrotors. In the considered case the upper bound of matched disturbances depends nonlinearly on the control itself. The closed-loop system stability is ensured for a selected maximum admissible value of the wind speed. The proposed in this article control strategy can be equipped with an additional wind estimator algorithm, as in [1], reducing automatically the control effort on the rotors when it is not necessary.

The article outline is as follows. The considered model of UAV is given in Section II. The control and disturbance bounds are calculated in Section III. The control design is presented in Section IV. The results of simulation are shown in Section V. The remarks and discussion conclude the paper in Section VI. 


\section{QUADROTOR DYNAMICAL MODEL}

This section presents the model of the UAV dynamics, which has configuration as it is shown in Fig. 1, and which is used for the Onera project to estimate the wind velocity, making the drone as a wind sensor.

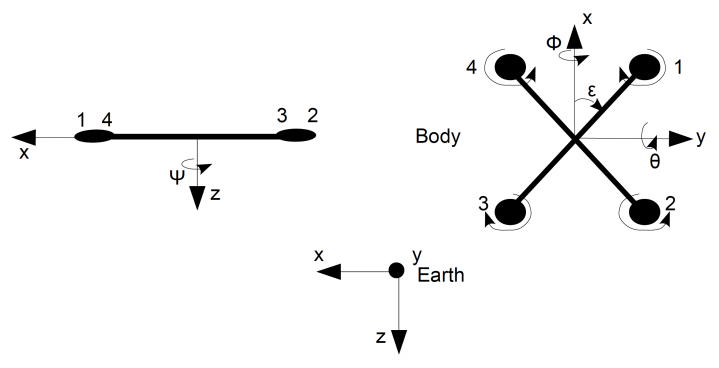

Fig. 1. UAV configuration

According to the identification work at low speed in [14] using the Parrot AR Drone 2.0, rotors gyroscopic effects and inertial counter torques are neglected since they are rather small.

The translation dynamics of the drone in the body frame yield

$$
m \dot{\mathbf{u}}+m \varpi \times \mathbf{u}=\mathbf{F}_{\text {aero }}+m \mathbf{R}^{T} \mathbf{g},
$$

where $m$ is the mass of the UAV, $\mathbf{u}=[u v w]^{T}$ is its linear velocity expressed in body frame, $\varpi=\left[\begin{array}{ll}p & q \\ { }^{T}\end{array}\right]^{T}$ is its angular velocity in body frame, $\mathbf{F}_{\text {aero }}=\left[F_{\text {Xaero }} F_{\text {Yaero }} F_{\text {Zaero }}\right]^{T}$ is the vector of the external aerodynamic forces in body frame, $\mathbf{g}=\left[\begin{array}{lll}0 & 0 & g\end{array}\right]^{T}$ is the gravity acceleration in inertial frame, $\mathbf{R}$ is the rotational matrix defined as

$$
\mathbf{R}=\left[\begin{array}{ccc}
c_{\psi} c_{\theta} & -s_{\psi} c_{\phi}+c_{\psi} s_{\theta} s_{\phi} & s_{\phi} s_{\psi}+c_{\psi} s_{\theta} c_{\phi} \\
s_{\psi} c_{\theta} & c_{\psi} c_{\phi}+s_{\psi} s_{\theta} s_{\phi} & -c_{\psi} s_{\phi}+s_{\psi} s_{\theta} c_{\phi} \\
-s_{\theta} & c_{\theta} s_{\phi} & c_{\theta} c_{\phi}
\end{array}\right]
$$

where $c_{\psi}=\cos (\psi), s_{\psi}=\sin (\psi)$ and similarly.

The rotational dynamics of the drone with respect to inertial earth frame are

$$
\mathbf{I} \dot{\varpi}=-\varpi \times \mathbf{I} \varpi+\tau_{\text {aero }}
$$

where $\mathbf{I}$ is the inertia matrix of the UAV, and $\tau_{\text {aero }}=$ $\left[L_{\text {aero }} M_{\text {aero }} N_{\text {aero }}\right]^{T}$ is the external aerodynamic moments in the body frame.

The relation between angular velocities and Euler angles

$$
\begin{gathered}
\dot{\phi}=p+\tan \theta(q \sin \phi+r \cos \phi), \dot{\theta}=q \cos \phi-r \sin \phi, \\
\dot{\psi}=\frac{q \sin \phi+r \cos \phi}{\cos \theta},
\end{gathered}
$$

are considered avoiding the singularities $\theta \neq \frac{\pi}{2}$, which is a reasonable assumption in our case since the topic of this article is not to achieve aggressive maneuvers.

Hence the full model of the system is presented by the equations (1), (2), (3).

\section{A. Aerodynamics}

The aerodynamic forces, moments, and coefficients are derived using a combination of momentum and blade element theory in helicopters, well explained by [15], [16], [17].

Aerodynamic forces and moments for each rotor, where subscript $j$ indicates the $j^{\text {th }}$ rotor, are derived as

$$
\begin{aligned}
F_{X j} & =-\rho A R^{2} \frac{u_{j}-u_{w}}{\sqrt{\left(u_{j}-u_{w}\right)^{2}+\left(v_{j}-v_{w}\right)^{2}}} C_{H j} \omega_{j}^{2}, \\
F_{Y j} & =-\rho A R^{2} \frac{v_{j}-v_{w}}{\sqrt{\left(u_{j}-u_{w}\right)^{2}+\left(v_{j}-v_{w}\right)^{2}}} C_{H j} \omega_{j}^{2}, \\
F_{Z_{j}} & =-\rho A R^{2} C_{T_{j}} \omega_{j}^{2}, \\
L_{j} & =-\operatorname{sign} \omega_{j} \rho A R^{3} \frac{u_{j}-u_{w}}{\sqrt{\left(u_{j}-u_{w}\right)^{2}+\left(v_{j}-v_{w}\right)^{2}}} C_{R m j} \omega_{j}^{2}, \\
M_{j} & =-\operatorname{sign} \omega_{j} \rho A R^{3} \frac{v_{j}-v_{w}}{\sqrt{\left(u_{j}-u_{w}\right)^{2}+\left(v_{j}-v_{w}\right)^{2}}} C_{R m j} \omega_{j}^{2}, \\
N_{j} & =-\operatorname{sign} \omega_{j} \rho A R^{3} C_{Q_{j}} \omega_{j}^{2},
\end{aligned}
$$

where $\rho$ is the air density, $A$ is the rotor area, $R$ is the rotor radius, $\left[u_{w} v_{w} w_{w}\right]$ is the wind velocity with respect to the earth in body frame, $C_{H}$ is the hub force coefficient, $C_{T}$ is rotor thrust coefficient, $\omega$ is the rotor angular speed, $C_{Q}$ is the rotor drag moment coefficient, $C_{R m}$ is the rotor rolling moment coefficient.

Total aerodynamic forces are

$$
F_{\text {Xaero }}=\sum_{j=1}^{4} F_{X j}, F_{\text {Yaero }}=\sum_{j=1}^{4} F_{Y j}, F_{\text {Zaero }}=\sum_{j=1}^{4} F_{Z_{j}} .
$$

Total aerodynamic moments are

$$
\begin{gathered}
L_{\text {aero }}=\sum_{j=1}^{4}\left(L_{j}+F_{Z_{j}} l \mathrm{~s}_{j}-h F_{Y j}\right), \\
M_{\text {aero }}=\sum_{j=1}^{4}\left(M_{j}-F_{Z_{j}} l \mathrm{c}_{j}+h F_{X_{j}}\right) \\
N_{\text {aero }}=\sum_{j=1}^{4}\left(N_{j}+F_{Y_{j}} l \mathrm{c}_{j}-F_{X_{j}} l \mathrm{~s}_{j}\right),
\end{gathered}
$$

where $h$ is the distance between rotors plane and the center of gravity of the UAV, $l$ is the arm lenght, and with $\mathrm{c}_{j}=\cos \left(\frac{\pi}{2}(j-1)+\varepsilon\right), \mathrm{s}_{j}=\sin \left(\frac{\pi}{2}(j-1)+\varepsilon\right)$. In our UAV configuration we have $\varepsilon=\frac{\pi}{4}$, thus for vectors $\mathrm{c}_{j}$ and $\mathrm{s}_{j}$ we have cosines and sinus of the angles $\left[\frac{\pi}{4}, \frac{3}{4} \pi, \frac{5}{4} \pi, \frac{7}{4} \pi\right]$.

According to [14] and thanks to experiments on Parrot drone, the aerodynamic coefficients are identified as follows

$$
\begin{gathered}
C_{T j}=C_{T s t a t}+K_{z} \frac{w_{j}-w_{w}}{R\left|\omega_{j}\right|}, \quad C_{T \text { stat }}>0 \\
\lambda_{j}=\lambda_{\text {stat }}-\frac{4}{\sigma a} K_{z} \frac{w_{j}-w_{w}}{R\left|\omega_{j}\right|}, \quad K_{z} \geq 0, \lambda_{\text {stat }}>0 \\
\mu_{j}=\frac{1}{\left|\omega_{j}\right| R} \sqrt{\left(u_{j}-u_{w}\right)^{2}+\left(v_{j}-v_{w}\right)^{2}}, C_{R m j}=\sigma a \frac{\mu_{j}}{8}\left(\lambda_{j}-\frac{4}{3}\right), \\
C_{Q_{j}}=\frac{\sigma C_{D 0}}{8}\left(1+\mu_{j}^{2}\right)+\sigma a \lambda_{j}\left(\frac{\theta_{0}}{6}-\frac{\lambda_{j}}{4}\right), \\
C_{H j}=K_{D} \mu_{j}, \quad K_{D} \geq 0 .
\end{gathered}
$$


where $\sigma$ is the rotor solidity ratio, $a$ is the lift curve slope of the blade section, $C_{D 0}$ is the drag coefficient of the blade section, $\theta_{0}$ is the angle of attack of the root profile, $\lambda$ is the inflow ratio, $\mu$ is the advance ratio, subscript stat indicates the value in stationary phase. The shape of above UAV coefficients can be explained recalling aerodynamic science: the thrust is the resultant of the vertical forces acting on all the blade elements. The inflow ratio is the ratio between the component of UAV velocity perpendicolar to the rotor disk with respect to the blade tip speed. The advance ratio indicates the component of the UAV velocity parallel to the rotor disk with respect to the blade tip speed. The rolling moment of a propeller exists in forward flight when the advancing blade is producing more lift than the retreating one and it is the integration over the entire rotor of the lift of each section acting at a given radius. The hub forces is the resultant of the horizontal forces acting on all the blade elements. The drag moment about the rotor shaft is caused by the aerodynamic forces acting on the blade elements, the horizontal forces acting on the rotor are multiplied by the moment arm and integrated over the rotor.

\section{Control System EQUATIONS}

The model presented in previous section can be rewritten in state-space form $\dot{X}=f(X, U, d)$, where $U=\left[U_{z} U_{\theta} U_{\phi} U_{\psi}\right]^{T}$ is the control input, disturbances $d$ are described in the next subsection, and the state vector $X$ is chosen as $X=$ $[z, \dot{z}, \phi, \theta, \psi, p, q, r]^{T}$ The relations between the control inputs and the rotor velocities are defined by an invertible matrix

$$
\left[\begin{array}{c}
U_{z} \\
U_{\theta} \\
U_{\phi} \\
U_{\psi}
\end{array}\right]=\left[\begin{array}{cccc}
K_{f} & K_{f} & K_{f} & K_{f} \\
K_{f} l \mathrm{c}_{j} & K_{f} l \mathrm{c}_{j} & K_{f} l \mathrm{c}_{j} & K_{f} l \mathrm{c}_{j} \\
-K_{f} l \mathrm{~s}_{j} & -K_{f} l \mathrm{~s}_{j} & -K_{f} l \mathrm{~s}_{j} & -K_{f} l \mathrm{~s}_{j} \\
K_{m} & -K_{m} & K_{m} & -K_{m}
\end{array}\right]\left[\begin{array}{c}
\omega_{1}^{2} \\
\omega_{2}^{2} \\
\omega_{3}^{2} \\
\omega_{4}^{2}
\end{array}\right],
$$

with $\omega_{\min } \leq \omega_{j} \leq \omega_{\max }$, where $K_{f}=\rho A R^{2} C_{T \text { stat }}$ and $K_{m}=$ $\rho A R^{3}\left(\frac{\sigma C_{D 0}}{8}+\lambda_{\text {stat }} \sigma a\left(\frac{\theta_{0}}{6}-\frac{\lambda_{\text {stat }}}{4}\right)\right)$. In this work, the control inputs are selected to be proportional to the terms with $\omega_{j}^{2}$. Thus, expanding (1) and (2), the other terms dependent linearly on $\omega_{j}$ and wind velocities are considered as disturbances. Since we do not know in advance the wind perturbations, then we cannot use these terms in controls. Such a decomposition of thrust (which is proportional to $\omega_{j}$ and $\omega_{j}^{2}$ ) and selection of disturbances are almost exact in the hover flight, where we have that $(p, q, r) \approx(\dot{\phi}, \dot{\theta}, \dot{\psi})$.

\section{A. Definition of disturbance upper bounds}

In literature, fixed bounds are often assumed on each component of the disturbance input vector $d$, which means $|d| \leq D$ for some known $D \geq 0$. However, this is often quite conservative. In our case the varying state-dependent bounds will be considered, but we will assume boundedness of the wind velocities: $\left|u_{w}\right| \leq D_{x},\left|v_{w}\right| \leq D_{y},\left|w_{w}\right| \leq D_{z}$, for some known $D_{x} \geq 0, D_{y} \geq 0, D_{z} \geq 0$ which come out from the on-board wind estimator explained in [1]. Taking into account the proposed model equations we selected $d=\left[d_{z}, d_{\phi}, d_{\theta}, d_{\psi}\right]$, substituting (6), (4) and (5) in (1) and (2), and considering the terms not proportional to $\omega_{j}^{2}$.

To design a control, which is able to compensate the disturbances, we have to evaluate the upper bounds for them.

1) Disturbance in $z$ dynamics: From the computed $d_{z}$

$$
\begin{gathered}
\left|d_{z}\right|=\left|\tilde{K}_{z} \sum_{j=1}^{4}\left(w_{j}-w_{w}\right) \leq \tilde{K}_{z} \sum_{j=1}^{4}\right| w_{j}-w_{w}|| \omega_{j} \mid \\
\leq \tilde{K}_{z} \sum_{j=1}^{4}\left(\left|w_{j}\right|+\left|w_{w}\right|\right)\left|\omega_{j}\right| \leq \tilde{K}_{z}\left(\max _{j}\left|w_{j}\right|+\left|w_{w}\right|\right) \sum_{j=1}^{4}\left|\omega_{j}\right|,
\end{gathered}
$$

where $\tilde{K}_{z}=\rho A R K_{z}$. Using the control equation $U_{z}=$ $K_{f} \sum_{j=1}^{4} \omega_{j}^{2}$, from which applying the Jensen's inequality an upper estimate can be obtained

$$
\sum_{j=1}^{4}\left|\omega_{j}\right| \leq K \sqrt{\left|U_{z}\right|}, \quad K=\frac{2}{\sqrt{K_{f}}}
$$

An upper bound of the disturbance becomes

$$
\left|d_{z}\right| \leq \bar{K}_{z}\left(|X|+D_{z}\right) \sqrt{\left|U_{z}\right|}, \quad \bar{K}_{z}=K \rho A R K_{z} .
$$

2) Disturbance in roll dynamics: From the computed $d_{\phi}$ and (7), the disturbance becomes

$$
\left|d_{\phi}\right| \leq \tilde{K}_{\phi}\left(f_{\phi 1}(X)+D_{\phi 1}\right) \sqrt{\left|U_{z}\right|}+\bar{K}_{\phi}\left(f_{\phi 2}(X)+D_{\phi 2}\right),
$$

where $K_{\phi 1}=\rho A R^{2} \frac{\sigma a}{2}\left(\frac{\theta_{0}}{3}-\frac{\lambda_{\text {stat }}}{4}\right), \quad K_{\phi 2}=\rho A R h K_{D}, \quad K_{\phi 3}=$ $l K_{z} \rho A R, \quad \tilde{K}_{\phi}=K, f_{\phi 1}(X)=K_{\phi 1} \max _{j}\left|u_{j}\right|+$ $K_{\phi 2} \max _{j}\left|v_{j}\right|+K_{\phi 3} \max _{j}\left|w_{j} \mathrm{~s}_{j}\right|, \quad D_{\phi 1}=K_{\phi 1} D_{x}+$ $K_{\phi 2} D_{y}+K_{\phi 3} D_{z} \max _{j}\left|\mathrm{~s}_{j}\right|, \quad \bar{K}_{\phi}=\frac{1}{2} \rho A R K_{z}, \quad f_{\phi 2}(X)=$ $\max _{j}\left|u_{j}\right|^{2}+\max _{j}\left|w_{j}\right|^{2}, \quad D_{\phi 2}=D_{x}^{2}+D_{z}^{2}$.

3) Disturbance in pitch dynamics: From the computed $d_{\theta}$ and (7), the disturbance becomes

$$
\left|d_{\theta}\right| \leq \tilde{K}_{\theta}\left(f_{\theta 1}(X)+D_{\theta 1}\right) \sqrt{\left|U_{z}\right|}+\bar{K}_{\theta}\left(f_{\theta 2}(X)+D_{\theta 2}\right),
$$

where $K_{\theta 1}=\rho A R^{2} \frac{\sigma a}{2}\left(\frac{\theta_{0}}{3}-\frac{\lambda_{\text {stat }}}{4}\right), \quad K_{\theta 2}=\rho A R h K_{D}, \quad K_{\theta 3}=$ $l K_{z} \rho A R, \quad \tilde{K}_{\theta}=K, f_{\theta 1}(X)=K_{\theta 1} \max _{j}\left|v_{j}\right|+$ $K_{\theta 2} \max _{j}\left|u_{j}\right|+K_{\theta 3} \max _{j}\left|w_{j} \mathrm{c}_{j}\right|, \quad D_{\theta 1}=K_{\theta 1} D_{y}+$ $K_{\theta 2} D_{x}+K_{\theta 3} D_{z} \max _{j}\left|\mathrm{c}_{j}\right|, \quad \bar{K}_{\theta}=\frac{1}{2} \rho A R K_{z}, \quad f_{\theta 2}(X)=$ $\max _{j}\left|v_{j}\right|^{2}+\max _{j}\left|w_{j}\right|^{2}, \quad D_{\theta 2}=D_{y}^{2}+D_{z}^{2}$.

4) Disturbance in yaw dynamics: From the computed $d_{\psi}$ and (7), the disturbance becomes

$$
\left|d_{\psi}\right| \leq \tilde{K}_{\psi}\left(f_{\psi 1}(X)+D_{\psi 1}\right) \sqrt{\left|U_{z}\right|}+\bar{K}_{\psi}\left(f_{\psi 2}(X)+D_{\psi 2}\right),
$$

where $\quad K_{\psi 1}=\rho A R^{2} K_{z}\left(\frac{2 \theta_{0}}{3}-2 \lambda_{\text {stat }}\right), \quad K_{\psi 2}=$ $\rho A R l K_{D}, \quad K_{\psi 3}=\rho A R l K_{D}, \tilde{K}_{\psi}=K, f_{\psi 1}(X)=$ $K_{\psi 1} \max _{j}\left|w_{j}\right|+K_{\psi 2} \max _{j}\left|v_{j} \mathrm{c}_{j}\right|+K_{\psi 3} \max _{j}\left|u_{j} \mathrm{~s}_{j}\right|, \quad D_{\psi 1}=$ $K_{\psi 1} D_{z}+K_{\psi 2} D_{y} \max _{j}\left|\mathrm{c}_{j}\right|+K_{\psi 3} D_{x} \max _{j}\left|\mathrm{~s}_{j}\right| \bar{K}_{\psi}=$ $\rho A R, f_{\psi 2}(X)=\frac{\sigma C_{D 0}}{8}\left(\max _{j}\left|u_{j}\right|^{2}+\max _{j}\left|v_{j}\right|^{2}\right)+$ $\frac{4}{\sigma a} \max _{j}\left|w_{j}\right|^{2}, \quad D_{\psi 2}=\frac{\sigma C_{D 0}}{8}\left(D_{x}^{2}+D_{y}^{2}\right)+\frac{4}{\sigma a} D_{z}^{2}$.

\section{CONTROL DESIGN}

For synthesis of control law the SMC approach will be applied. This control methodology takes into account and compensates the matched disturbances. The big issue for the considered problem is that the disturbance $d$ depends on wind, the control itself, and state of the system, as shown above. Thus, a mild development of SMC approach is needed. 
To this end, the sliding surfaces in this work (for brevity of presentation) are selected proportional to the errors $e_{i}$

$$
S_{i}=\dot{e}_{i}+\alpha_{i} e_{i}, \quad \alpha_{i}>0,
$$

where $i \in(z, \phi, \theta, \psi)$. The Lyapunov function is chosen as

$$
V_{i}=\frac{1}{2} S_{i}^{2}
$$

\section{A. Altitude control}

The dynamics of $z$ can be rewritten in the earth frame

$$
\ddot{z}=g-(\cos \phi \cos \theta) \frac{1}{m}\left(U_{z}+d_{z}\right) .
$$

In hover state we have $\cos \theta \cos \phi \approx 1$, thus with a rotation of the UAV, a reasonable assumption is that $|\cos \phi \cos \theta| \geq \gamma>0$, where $\gamma$ is our operating point limit. To build the altitude control, the regulation error has been chosen as

$$
e_{z}=z-z_{d e s}
$$

where $z_{\text {des }}$ is the desired altitude for UAV. Thus, using (13) and its derivative in (10), the derivative of the sliding surface is $\dot{S}_{z}=\ddot{z}+\alpha_{z} \dot{z}$, which is, using (12), also equal to

$$
\dot{S}_{z}=g-\frac{\cos \theta \cos \phi}{m}\left(U_{z}+d_{z}\right)+\alpha_{z} \dot{z}
$$

The following expression of the control can be selected

$$
U_{z}=\frac{m}{\cos \theta \cos \phi}\left(g-\tilde{u}_{z}+\alpha_{z} \dot{z}\right)
$$

where $\tilde{u}_{z}$ is an auxiliary control defined later. After substitution of this control in (14), we obtain

$$
\dot{S}_{z}=\tilde{u}_{z}+d_{z} \frac{\cos \theta \cos \phi}{m} .
$$

Using the Lyapunov function (11) with (15), its derivative is

$$
\dot{V}=S_{z} \tilde{u}_{z}+S_{z} d_{z} \frac{\cos \theta \cos \phi}{m} \leq S_{z} \tilde{u}_{z}+\left|S_{z}\right|\left|d_{z} \frac{1}{m}\right| .
$$

Then, using (8), the Lyapunov derivative becomes $\dot{V} \leq$ $S_{z} \tilde{u}_{z}+\left|S_{z}\right| \frac{1}{m} \bar{K}_{z}\left(|X|+D_{z}\right) \sqrt{\left|U_{z}\right|}$. From the expression (IV-A) we can derive $\left|U_{z}\right| \leq \frac{m}{\gamma}\left(\left|g+\alpha_{z} \dot{z}\right|+\left|\tilde{u}_{z}\right|\right)$. Then, substituting in Lyapunov derivative, we obtain

$$
\dot{V} \leq S_{z} \tilde{u}_{z}+\left|S_{z}\right|\left(\rho(X)+v(X) \sqrt{\left|\tilde{u}_{z}\right|}\right),
$$

where $\rho(X)=\frac{1}{m} \sqrt{\frac{m}{\gamma}} \bar{K}_{z}\left(|X|+D_{z}\right) \sqrt{\left|g+\alpha_{z} \dot{z}\right|}, \quad v(X)=$ $\frac{1}{m} \sqrt{\frac{m}{\gamma}} \bar{K}_{z}\left(|X|+D_{z}\right)$. Let us look for the control in the form

$$
\tilde{u}_{z}=-\beta(X) \operatorname{sign}\left(S_{z}\right),
$$

where $\beta(X)$ is a function to synthesize. Substituting this control in the obtained inequality (16) we get $\dot{V} \leq\left|S_{z}\right|(\rho(X)+$ $v(X) \sqrt{\beta(X)}-\beta(X))$, and it is necessary to ensure by a choice of $\beta(X)$ that $\rho(X)+v(X) \sqrt{\beta(X)}-\beta(X)<0$. Solving this quadratic inequality with respect to $\beta(X)$ we found

$$
\beta(X)=\frac{1}{2}\left(v(X)^{2}+2 \rho(X)+v(X) \sqrt{v^{2}(X)+4 \rho(X)}\right)+\delta,
$$

where for tuning parameter $\delta>0$ the system state trajectory reaches and stays on the sliding surface $S_{z}$, which means asymptotic convergence of $e_{z}$ to the origin. Moreover, finitetime stability with respect to $S_{z}$ can be proved according to [4], since $\dot{V}<-\delta \sqrt{2 V}$.

\section{B. Attitude control}

In the following, $(\dot{\phi}, \dot{\theta}, \dot{\psi})$ are computed from (3).

1) Roll control: The dynamics of $\phi$ can be rewritten as

$$
\ddot{\phi}=\dot{\theta} \dot{\psi} \frac{I_{y y}-I_{z z}}{I_{x x}}+\frac{1}{I_{x x}}\left(U_{\phi}+d_{\phi} .\right)
$$

To build the roll control, the error has been chosen as

$$
e_{\phi}=\phi-\phi_{d e s}
$$

where $\phi_{\text {des }}$ is the desired roll angle. Thus, using (18) and its derivative in (10), let us calculate the derivative of the sliding surface $\dot{S}_{\phi}=\ddot{\phi}+\alpha_{\phi} \dot{\phi}$, which is, using (17), also equal to

$$
\dot{S}_{\phi}=\dot{\theta} \dot{\psi} \frac{I_{y y}-I_{z z}}{I_{x x}}+\frac{1}{I_{x x}}\left(U_{\phi}+d_{\phi}\right)+\alpha_{\phi} \dot{\phi} .
$$

The following expression of the control can be selected

$$
U_{\phi}=I_{x x}\left(-\dot{\theta} \dot{\psi} \frac{I_{y y}-I_{z z}}{I_{x x}}+\tilde{u}_{\phi}-\alpha_{\phi} \dot{\phi}\right) .
$$

After substitution of this control in (19), we obtain

$$
\dot{S}_{\phi}=\tilde{u}_{\phi}+d_{\phi} \frac{1}{I_{x x}} .
$$

Using the Lyapunov function (11) with (20) and (9), its derivative becomes

$$
\begin{aligned}
& \dot{V}=S_{\phi} \tilde{u}_{\phi}+S_{\phi} d_{\phi} \leq S_{\phi} \tilde{u}_{\phi}+\left|S_{\phi}\right|\left|d_{\phi} \frac{1}{I_{x x}}\right| \leq S_{\phi} \tilde{u}_{\phi}+\frac{\left|S_{\phi}\right|}{I_{x x}} \\
& \times\left(\tilde{K}_{\phi}\left(f_{\phi 1}(X)+D_{\phi 1}\right) \sqrt{\left|U_{z}\right|}+\bar{K}_{\phi}\left(f_{\phi 2}(X)+D_{\phi 2}\right)\right) .
\end{aligned}
$$

To have (21) negative, the auxiliary control for $\phi$ dynamics must be

$$
\begin{gathered}
\tilde{u}_{\phi}=-\frac{1}{I_{x x}} \operatorname{sign} S_{\phi}\left(\tilde{K}_{\phi}\left(f_{\phi 1}(X)+D_{\phi 1}\right) \sqrt{\left|U_{z}\right|}\right. \\
\left.+\bar{K}_{\phi}\left(f_{\phi 2}(X)+D_{\phi 2}\right)\right) .
\end{gathered}
$$

Pitch and yaw controls can be designed following computations similar to the roll one. So, for brevity of presentation, only final expressions are given below.

2) Pitch control: The auxiliary control for $\theta$ dynamics

$$
\begin{gathered}
\tilde{u}_{\theta}=-\frac{1}{I_{y y}} \operatorname{sign}_{\theta}\left(\tilde{K}_{\theta}\left(f_{\theta 1}(X)+D_{\theta 1}\right) \sqrt{\left|U_{z}\right|}\right. \\
\left.+\bar{K}_{\theta}\left(f_{\theta 2}(X)+D_{\theta 2}\right)\right),
\end{gathered}
$$

with the following expression of control

$$
U_{\theta}=I_{y y}\left(-\dot{\phi} \dot{\psi} \frac{I_{z z}-I_{x x}}{I_{y y}}+\tilde{u}_{\theta}-\alpha_{\theta} \dot{\theta}\right) .
$$

3) Yaw control: The auxiliary control for $\psi$ dynamics

$$
\begin{gathered}
\tilde{u}_{\psi}=-\frac{1}{I_{z z}} \operatorname{sign} S_{\psi}\left(\tilde{K}_{\psi}\left(f_{\psi 1}(X)+D_{\psi 1}\right) \sqrt{\left|U_{z}\right|}\right. \\
\left.+\bar{K}_{\psi}\left(f_{\psi 2}(X)+D_{\psi 2}\right)\right),
\end{gathered}
$$

with the following expression of control

$$
U_{\psi}=I_{z z}\left(-\dot{\theta} \dot{\phi} \frac{I_{x x}-I_{y y}}{I_{z z}}+\tilde{u}_{\psi}-\alpha_{\psi} \dot{\psi}\right) \text {. }
$$




\section{Chattering reduction}

Unfortunately a big SMC issue is the chattering of the control which can ruin the rotors. This phenomenum is caused by the presence of a discontinuous function in the control and due to the unmodelled dynamics or uncertainty of the UAV model parameters. In literature the chattering is a standard and well-known issue, discussed in many articles such as [5], and plenty sophisticated methods to avoid it have been presented. A saturation function is frequently used in implementation as the simplest tool to suppress chattering, when a smooth nonlinearity replaces the sign function to avoid the discontinuity at the origin in order to obtain a smoother behavior:

$$
\operatorname{sat}_{\xi}(x)= \begin{cases}\operatorname{sign}(x) & \text { if }|x|>\xi \\ \arctan \left(\frac{1}{\xi} x\right) & \text { otherwise }\end{cases}
$$

with $\xi>0$. Moreover, according to [18], if for a sign function all trajectories converge to an equilibrium, then with a saturation, for a sufficiently small $\xi$, all trajectories converge to a compact set $\Omega_{\xi}$ around that equilibrium. Thus, saturation functions are a standard tool for chattering reduction in SMC that leads to a practical stability in the closed-loop system. In our case $\xi$ should be chosen small enough to find a compromise between chattering reduction and minimal acceptable error.

Another way to reduce chattering consists in application of a high order SMC, an example of such a recent tool is the quasi-continuous control [19], which can also be considered as an approximation of the sign on the plane. Here, the sliding surface is not useful anymore since the control allows the reference point to be reached by the state directly from any initial condition in a finite time without any auxiliary dynamics.

Recall that for $z$ dynamics, the second derivative of the position error has the form

$$
\ddot{e}_{z}=g-\frac{\cos \theta \cos \phi}{m}\left(U_{z}+d_{z}\right) .
$$

In [20],[19] it is stated that for the system

$$
\ddot{e}_{z}=-\beta_{q}(X) \frac{\left\lceil\dot{e}_{z}\right\rfloor^{2}+e_{z}}{\left|\dot{e}_{z}\right|^{2}+\left|e_{z}\right|}+d_{z},
$$

where $\beta_{q}(X)$ is the new function to synthesize, the origin $e=\dot{e}=0$ is reached in a finite time provided that $\beta_{q}(X)>$ $\left\|d_{z}\right\|_{\infty}$. Thus, substituting (23) in (22) the quasi-continuous control in our case has to be selected as follows

$$
U_{z}=\frac{m}{\cos \phi \cos \theta}\left(g+\beta_{q}(X) \frac{\left\lceil\dot{e}_{z}\right\rfloor^{2}+e_{z}}{\left|\dot{e}_{z}\right|^{2}+\left|e_{z}\right|}\right) .
$$

The control is bounded by $\left|U_{z}\right| \leq \frac{m}{\gamma}\left(g+\beta_{q}(X)\right)$, and taking into account the derived bound for $d_{z}$ the final inequality for $\beta_{q}$ is $\beta_{q}(X)>\left(\bar{K}_{z}(|X|)+D_{z}\right) \sqrt{\frac{m}{\gamma}\left(g+\beta_{q}(X)\right)}$, which has a solution

$$
\begin{gathered}
\beta_{q}(X)>\frac{\bar{K}_{z}(|X|)+D_{z}}{2 \gamma}\left[m\left(\bar{K}_{z}(|X|)+D_{z}\right)\right. \\
\left.+\sqrt{4 \gamma g m+m^{2}\left(\bar{K}_{z}(|X|)+D_{z}\right)^{2}}\right] .
\end{gathered}
$$

The other dynamics can be designed following similar computations. So, for brevity of presentation, only final expressions are given below for roll, pitch and yaw

$$
\begin{aligned}
U_{\phi}= & -\dot{\theta} \dot{\psi}\left(I_{y y}-I_{z z}\right)-\frac{\left\lceil\left.\dot{e}_{\phi}\right|^{2}+e_{\phi}\right.}{\left|\dot{e}_{\phi}\right|^{2}+\left|e_{\phi}\right|}\left(\tilde{K}_{\phi}\left(f_{\phi 1}(X)+D_{\phi 1}\right)\right. \\
& \left.\times \sqrt{\left|U_{z}\right|}+\bar{K}_{\phi}\left(f_{\phi 2}(X)+D_{\phi 2}\right)\right), \\
U_{\theta}= & -\dot{\phi} \dot{\psi}\left(I_{z z}-I_{x x}\right)-\frac{\left\lceil\left.\dot{e}_{\theta}\right|^{2}+e_{\theta}\right.}{\left|\dot{e}_{\theta}\right|^{2}+\left|e_{\theta}\right|}\left(\tilde{K}_{\theta}\left(f_{\theta 1}(X)+D_{\theta 1}\right)\right. \\
& \left.\times \sqrt{\left|U_{z}\right|}+\bar{K}_{\theta}\left(f_{\theta 2}(X)+D_{\theta 2}\right)\right), \\
U_{\psi}= & -\dot{\theta} \dot{\phi}\left(I_{x x}-I_{y y}\right)-\frac{\left\lceil\left.\dot{e}_{\psi}\right|^{2}+e_{\psi}\right.}{\left|\dot{e}_{\psi}\right|^{2}+\left|e_{\psi}\right|}\left(\tilde{K}_{\psi}\left(f_{\psi 1}(X)+D_{\psi 1}\right)\right. \\
& \left.\times \sqrt{\left|U_{z}\right|}+\bar{K}_{\psi}\left(f_{\psi 2}(X)+D_{\psi 2}\right)\right) .
\end{aligned}
$$

To avoid completely the chattering a slight modification of the quasi-continuous control can be introduced: adding a small constant in the denominator.

\section{NUMERICAL EXPERIMENTS}

The UAV dynamics and controls have been implemented in Matlab/Simulink environment. To show the performance of the proposed control laws with respect to reference input signals, we are going to consider the Parrot Drone parameters which has mass $m=0.47 \mathrm{~kg}$, rotors velocities $200 \mathrm{rad} / \mathrm{s} \leq$ $\omega_{j} \leq 400 \mathrm{rad} / \mathrm{s}$. Even if the control law is designed using some simplifying hypothesis, the final control is tested on a more complex UAV model which considers all the coefficient nonlinearities, validated through indoor experiments with the Parrot at the ONERA Aerodynamics, Aeroelasticity, Acoustics Department to simulate the real quadrotor behaviour. For simplicity of demonstration the desired references for the states and wind maximal speed are selected constant.

A flight case is illustrated which considers forward, lateral and vertical flights (coupling together $z, \phi, \theta, \psi)$. The corresponding disturbances and wind are illustrated in Fig. 2. The controls together with state variables and reference states are illustrated in figures 3, 4, where conventional first order SMC (conv-SMC), having the constant gain proportional to the sign functions $\left(K_{i}>\left\|d_{i}\right\|_{\infty}, i=z, \phi, \theta, \psi\right)$, the proposed first order SMC (1-SMC) and the high order quasi-continuous SMC (qc-SMC) are compared. In the proposed stabilizing algorithms, the control gains are functions of the state and wind velocity, then using a wind estimator the wind velocities can be substituted on-line in the control law. In this way, by adapting the control amplitude, the regulator effort on the rotors can be reduced when it is possible. The SMCs, which use the indicated gain functions, are less subjected to use high amplitude control, as we can conclude from these figures.

As a result, the presented simulations demonstrate the stability of the system affected by wind velocity and realistic external disturbances, respecting the limitations of the mini drone mass and thrust. 


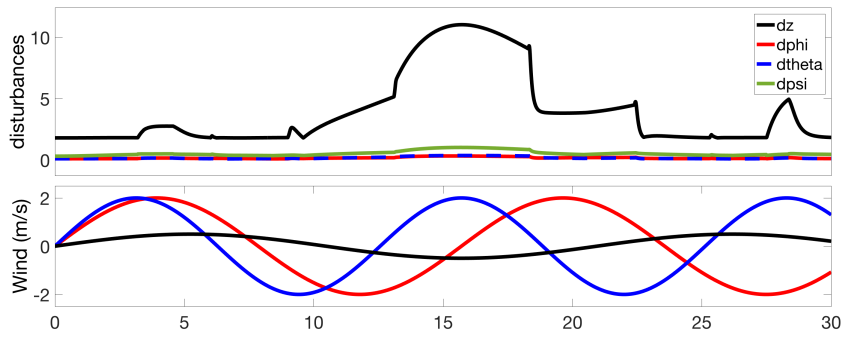

Fig. 2. Wind and disturbances. (—: wind $\mathrm{x},-$ : wind $\mathrm{y}, \mathrm{-}$ - : wind $\mathrm{z}$ ).

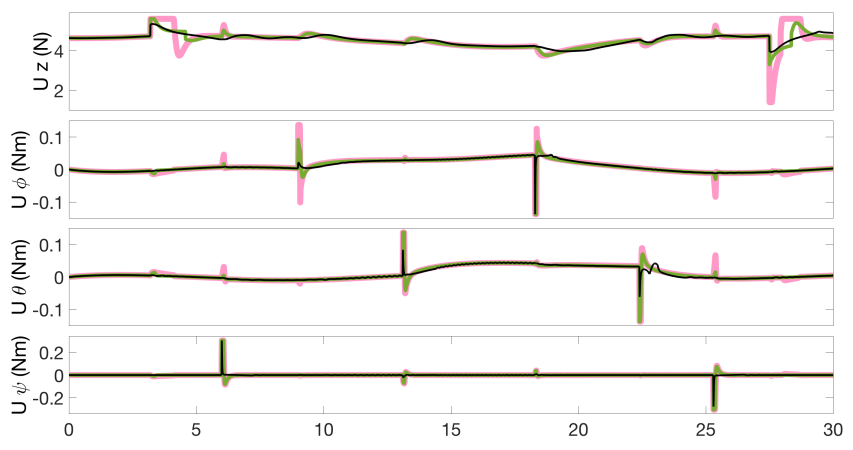

Fig. 3. Control signals. (-: conv-SMC, - : 1-SMC, -: qc-SMC).

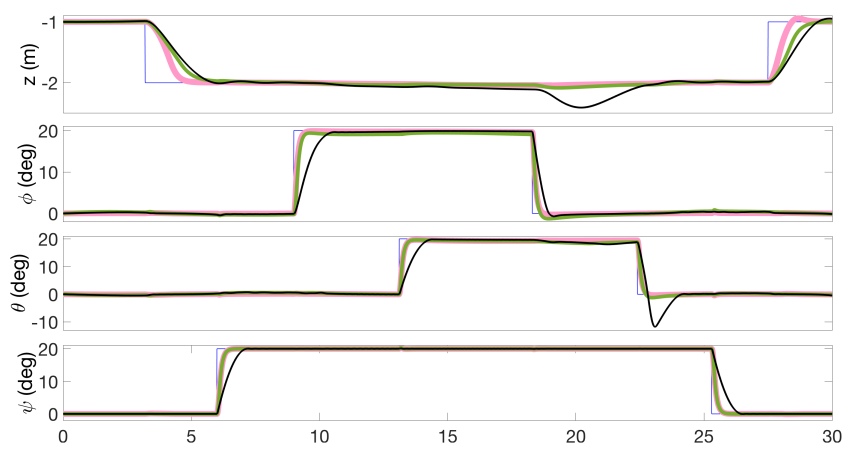

Fig. 4. State variables. (—: reference, - : conv-SMC, - : 1-SMC, qc-SMC).

\section{CONCLUSION}

A robust SMC approach is introduced to stabilize a small quadrotor UAV under wind perturbations. The constant gain, in conventional SMCs, is replaced with a varying function which depends on the wind speed, the control itself and the state. In this way we aim to reduce as much as possible the control effort on the rotors, adapting in real-time the weighted function used in the control design. The presented simulation results confirm the effectiveness of the designed control to stabilize the drone under varying wind, and its stability can always be proved changing the maximal allowed wind values. The contribution with respect to conventional first order SMC is presented, and a recent quasi-continuous high order SMC is also tested and verified. For a further investigation the following directions can be chosen: application of this control methodology using $x, y, z$ position way-points, the extension of the flight domain considering smooth trajectories with first and second derivatives, the coupling wind estimator and
SMC can be analyzed, and introduction of the rotor transfer functions and investigation their influence.

\section{REFERENCES}

[1] G. Perozzi, D. Efimov, J.-M. Biannic, L. Planckaert, and P. Coton, "Wind estimation algorithm for quadrotors using detailed aerodynamic coefficients," Submitted.

[2] N. Sydney, B. Smyth, and D. A. Paley, "Dynamic control of autonomous quadrotor flight in an estimated wind field," in 52nd IEEE Conference on Decision and Control, pp. 3609-3616, Dec. 2013. DOI: 10.1109/CDC.2013.6760438.

[3] S. Bouabdallah and R. Siegwart, "Full control of a quadrotor," in 2007 IEEE/RSJ International Conference on Intelligent Robots and Systems, pp. 153-158, Oct. 2007. DOI: 10.1109/IROS.2007.4399042.

[4] E. Bernuau, D. Efimov, W. Perruquetti, and A. Polyakov, "On homogeneity and its application in sliding mode control," Journal of the Franklin Institute, vol. 351, pp. 1866-1901, Apr. 2014. DOI: 10.1016/j.jfranklin.2014.01.007.

[5] I. González, S. Salazar, and R. Lozano, "Chattering-Free Sliding Mode Altitude Control for a Quad-Rotor Aircraft: Real-Time Application," Journal of Intelligent \& Robotic Systems, vol. 73, pp. 137-155, Jan. 2014. DOI: $10.1007 / \mathrm{s} 10846-013-9913-8$.

[6] T. Madani and A. Benallegue, "Backstepping Sliding Mode Control Applied to a Miniature Quadrotor Flying Robot," in IECON 2006 32nd Annual Conference on IEEE Industrial Electronics, pp. 700-705, Nov. 2006. DOI: 10.1109/IECON.2006.347236.

[7] S. Zeghlache, D. Saigaa, K. Kara, A. Harrag, and A. Bouguerra, "Backstepping sliding mode controller improved with fuzzy logic: Application to the quadrotor helicopter," Archives of Control Sciences, vol. 22, no. 3, pp. 315-342, 2012. DOI: 10.2478/v10170-011-0027-x.

[8] L. Derafa, A. Benallegue, and L. Fridman, "Super twisting control algorithm for the attitude tracking of a four rotors UAV," Journal of the Franklin Institute, vol. 349, pp. 685-699, Mar. 2012. DOI: 10.1016/j.jfranklin.2011.10.011.

[9] S. Rajappa, C. Masone, H. H. Bülthoff, and P. Stegagno, "Adaptive Super Twisting Controller for a quadrotor UAV," in 2016 IEEE International Conference on Robotics and Automation (ICRA), pp. 29712977, May 2016. DOI: 10.1109/ICRA.2016.7487462.

[10] E.-H. Zheng, J.-J. Xiong, and J.-L. Luo, "Second order sliding mode control for a quadrotor UAV," ISA Transactions, vol. 53, pp. 1350-1356, July 2014. DOI: 10.1016/j.isatra.2014.03.010.

[11] A. Benallegue, A. Mokhtari, and L. Fridman, "High-order slidingmode observer for a quadrotor UAV," International Journal of Robust and Nonlinear Control, vol. 18, pp. 427-440, Mar. 2008. DOI: $10.1002 /$ rnc. 1225 .

[12] Y. Zhang and A. Chamseddine, "Fault Tolerant Flight Control Techniques with Application to a Quadrotor UAV Testbed," in Automatic Flight Control Systems - Latest Developments, Dr. Thomas Lombaerts (Ed.), 2012. ISBN: 978-953-307-816-8. InTech.

[13] H. Bouadi, S. S. Cunha, A. Drouin, and F. Mora-Camino, "Adaptive sliding mode control for quadrotor attitude stabilization and altitude tracking," in 2011 IEEE 12th International Symposium on Computational Intelligence and Informatics (CINTI), pp. 449-455, Nov. 2011. DOI: 10.1109/CINTI.2011.6108547.

[14] L. Planckaert and P. Coton, "Quadrotor UAV aerodynamic model identification using indoor flight experiment and feasibility of UAV as wind gust sensor," in International Micro Air Vehicles Conference and Flight Competition IMAV 2015, (Aachen, Germany), Sept. 2015.

[15] G. J. Leishman, Principles of Helicopter Aerodynamics with CD Extra. Cambridge University Press, Apr. 2006. ch 2-5.

[16] W. Johnson, Helicopter Theory. Courier Corporation, Mar. 2012. ch $2-5,9-13,15$

[17] A. R. S. Bramwell, D. Balmford, and G. Done, Bramwell's Helicopter Dynamics, second edition. Butterworth-Heinemann, Apr. 2001. ch 2-7.

[18] H. K. Khalil, Nonlinear Systems. Prentice Hall, 2002.

[19] S. Ding, A. Levant, and S. Li, "Simple homogeneous sliding-mode controller," Automatica, vol. 67, pp. 22-32, May 2016. DOI: 10.1016/j.automatica.2016.01.017.

[20] G. Perozzi, D. Efimov, J.-M. Biannic, L. Planckaert, and P. Coton, "Wind rejection via quasi-continuous sliding mode technique to control safely a mini drone," in 7th European Conference for Aeronautics and Space Science (EUCASS), (Milan, Italy), July 2017. Available on: https://hal.inria.fr/hal-01566857. 